\title{
Comparative Analysis and Rational Design of dCas9- VP64 Variants for CRISPR Activation
}

\author{
Kohei Omachi' ${ }^{1}$ and Jeffrey H. Miner ${ }^{*}$
}

1Division of Nephrology, MSC 8126-012-0853, Washington University School of Medicine, 4523 Clayton Ave., St. Louis, Missouri, 63110, United States.

${ }^{*}$ Correspondence: Jeffrey H. Miner, E-mail: minerj@wustl.edu

\begin{abstract}
CRISPR/Cas9-mediated transcriptional activation (CRISPRa) is a powerful tool for investigating complex biological phenomena. Although CRISPRa approaches based on VP64 have been widely studied in both cultured cells and in animal models and exhibit great versatility for various cell types and developmental stages in vivo, different dCas9-VP64 versions have not been rigorously compared. Here, we compared different dCas9-VP64 constructs in identical contexts, including the cell lines used and the transfection conditions, for their ability to activate endogenous and exogenous genes. Moreover, we investigated the optimal approach for VP64 addition to VP64- and p300-based constructs. We found that MS2-MCP-scaffolded VP64 enhanced dCas9-VP64 and dCas9-p300 activity better than did direct VP64 fusion to the N-terminus of dCas9. dCas9VP64+MCP-VP64 and dCas9-p300+MCP-VP64 were superior to VP64-dCas9-VP64 for all target genes tested. Furthermore, multiplexing gRNA expression with dCas9-VP64+MCP-VP64 or dCas9-p300+MCP-VP64 significantly enhanced endogenous gene activation to a level comparable to CRISPRa-SAM with a single gRNA. Our findings demonstrate improvement of the dCas9-VP64 CRISPRa system and contribute to development of a versatile, efficient CRISPRa platform.
\end{abstract}

KEYWORDS: CRISPR activation, transcription factors, overexpression, epigenetic modification RUNNING TITLE: VP64-based CRISPR activation

\section{INTRODUCTION}

CRISPR/Cas9-mediated activation (CRISPRa)-based regulation of gene expression is a powerful tool for understanding complex biological phenomena, because it allows for the simultaneous regulation of multiple genes. CRISPRa has been used in broad fields of research, including direct cell reprogramming by controlling master transcription factors that regulate cell lineage, ${ }^{1-3}$ cancer modeling by activating oncogenes ${ }^{4}$, and therapeutic approaches by activating disease-modifying genes ${ }^{5}$ and genes deficient due to haploinsufficiency ${ }^{6}$.

The first generation version of CRISPRa, dCas9-VP64, was developed using VP64, a transcriptional activator, and dead Cas9 (dCas9) ${ }^{7}$, which has no nuclease activity, allowing activation of guide RNA (gRNA) -targeted endogenous genes ${ }^{8} 9$. Subsequently, protein tagging systems called Suntag (dCas9-Suntag-VP64) ${ }^{10}$ and MS2MCP (dCas9-VP64 + MCP-VP64) ${ }^{11}$ were developed to increase the number of VP64s at the same locus and enhance activation efficiency. In a similar approach, VP64-dCas9-VP64, in which one VP64 was added to the N-terminus of dCas9-VP64, exhibited increased efficiency of transcriptional activation ${ }^{1}$. More recently, additional transcriptional activators such as p65, Rta, and HSF1 have been identified, and CRISPRa-VPR ${ }^{2}, \mathrm{SAM}^{11}$,
SPH ${ }^{12}$ and TREE ${ }^{13}$ systems have been developed by combining multiple transcriptional activators. Based on a different concept from transcriptional inducers, dCas9 fused to epigenetic modifiers such as p300, histone acetylase ${ }^{14}$ and Tet1, a CpG DNA demethylase, ${ }^{15}$ have also been used to activate endogenous genes.

Although these studies have significantly contributed to the development of CRISPRa technology, most of them have been conducted in in vitro and ex vivo cell culture systems, and there are still challenges regarding in vivo applications. Specifically, several studies have reported on the in vivo toxicity of CRISPRa components. For example, it has been reported that VPR and SAM are toxic when highly expressed in Drosophila with a strong promoter ${ }^{16}$. Also, in mice, ubiquitous expression of VPR during development and expression in inhibitory neurons are toxic ${ }^{17}$. On the other hand, ubiquitous expression of Cas9 ${ }^{18}$ and dCas9-VP64 ${ }^{6}$ in mice is not overtly toxic, suggesting that neither dCas9 nor VP64 is toxic in vivo, and that high expression of either p65 or Rta, or both, may be responsible for the observed toxicity. Therefore, it is crucial to develop CRISPRa technologies that take into account both the efficiency of gene activation and side effects for in vivo applications. Based on these findings, we hypothesized that a simple VP64-based CRISPRa 


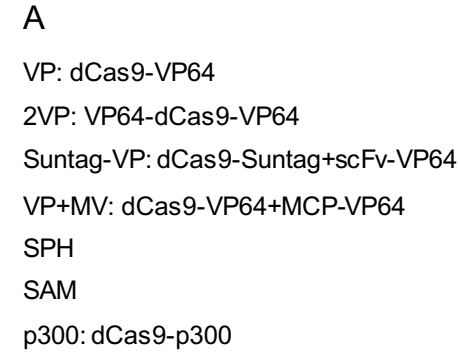

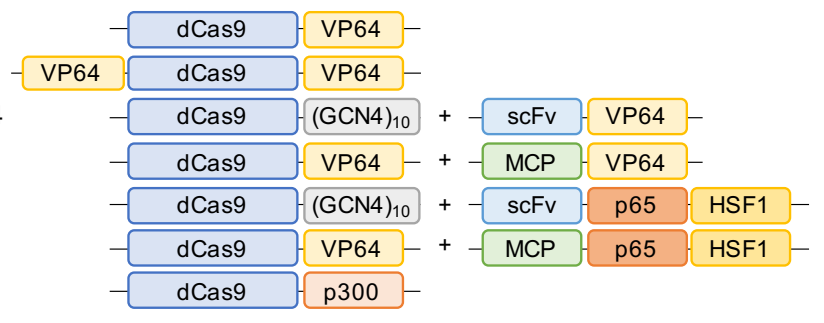
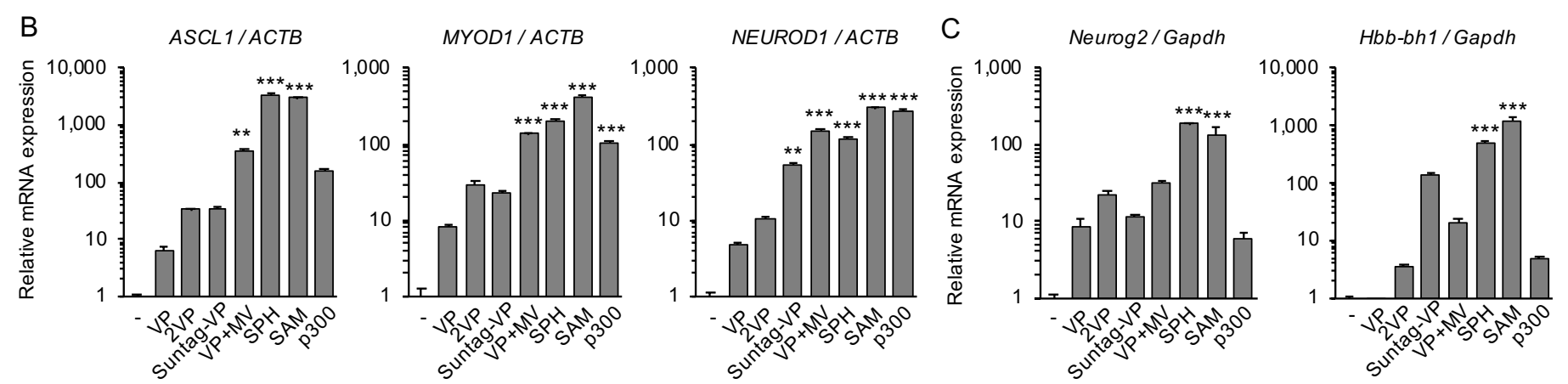

Figure 1. Functional comparison of various CRISPRa approaches to activate endogenous genes in human and mouse cells.

(A) Schematic diagrams of the CRISPRa systems used in the comparative study. (B) Expression analysis of three endogenous genes in human HEK293T cells. SAM and SPH induced the expression of ASCL1 and MYOD1 better than the other systems. Suntag-VP, VP+MV and p300 similarly activated ASCL1 and MYOD1, but p300 activated NEUROD1 stronger than the VP systems. (C) Expression analysis of two endogenous genes in mouse Neuro-2a cells. SAM and SPH induced both Neurog2 and Hbb-bhl better than the other systems did. For activation of both human and mouse genes, increasing the number of VP64s, as in 2VP, Suntag-VP, and $\mathrm{VP}+\mathrm{MV}$, improved the efficiency compared with VP. Error bars indicate the mean $\pm \mathrm{SE}(\mathrm{n}=3)$. Statistical analysis was performed using two-way ANOVA with Dunnett's multiple comparisons test. ${ }^{*}, P<0.05 ;{ }^{* *}, P<0.01 ;{ }^{* * *}, P<0.005$ vs. control.

would be useful in vivo in a variety of cell types, developmental stages, and pathological conditions. However, although there have been comparative studies of next-generation CRISPRa constructs such as SAM,

VPR, and SPH with high transcriptional activation capacity ${ }^{12,13,19-24}$, to the best of our knowledge there are not enough studies that seek to improve and characterize VP64-based CRISPRa by directly comparing activities in a systematic, controlled fashion. Here, we aimed to characterize and rationally design approaches for VP64based transcriptional activation of both endogenous and exogenous genes.

\section{RESULTS \\ Comparative analysis of CRISPR activation platforms}

To directly compare the ability of the established CRISPRa systems to activate transcription of endogenous and exogenous genes, we generated expression plasmids for dCas9 and domains recruiting the VP64 transcriptional activator by Suntag and gRNA scaffolding using the same promoter and backbone. The CRISPRaSPH ${ }^{12}$ and SAM ${ }^{11}$ systems were used as positive controls that promote high levels of transcriptional activation (Figure 1A). The degree of transcriptional activation of each system was assessed by quantifying increases in gene expression using previously validated human ASCL 1
${ }^{11}$, MYOD $^{11}$, and NEUROD1 ${ }^{19}$ and mouse Neurog2 ${ }^{11}$ and $H b b-b h{ }^{19}$ target gRNAs. Consistent with previous reports, the CRISPRa-SPH and -SAM systems were superior to all the VP64-based CRISPRa systems that we tested, in both human and mouse cells (Figure 1B, C). This suggests that VP64-based CRISPRa has room for optimization and improvement.

\section{N-terminal VP64 addition to dCas9-VP64}

Here, we focused on the second generation VP64-dCas9VP64 (2VP) ${ }^{1}$, dCas9-VP64+MCP-VP64 (VP+MV) ${ }^{11}$, and the epigenetic regulator dCas9-p300 (p300) ${ }^{14}$, which are more active than the first generation dCas9-VP64 (VP). Although dCas9-Suntag-VP64 ${ }^{10}$ was also better than VP, it was not included in this study due to limitations in combining it with other systems.

In agreement with previous reports, the present results (Figure 1B, C) showed that the addition of VP64 to the Nterminus of VP to make 2VP improves transcriptional induction. Therefore, in an attempt to improve VP+MV, we added VP64 to the N-terminus of dCas9 in VP+MV (Figure 2A, 2VP+MV). However, although 2VP $+\mathrm{MV}$ outperformed 2VP, it did not improve the ability of $\mathrm{VP}+\mathrm{MV}$ to activate $A S C L 1, M Y O D 1$ and NEUROD1 (Figure 2B). This result suggests that the number of VP64s recruited to the transcriptional start site targeted by a single gRNA is already sufficient for $\mathrm{VP}+\mathrm{MV}$, so there is no benefit from adding an N-terminal VP64 to VP+MV. 


\section{A}

2VP: VP64-dCas9-VP64

VP64 dCas9 VP64-

VP+MV: dCas9-VP64+MCP-VP64

dCas9 VP64 + MCP VP64

2VP+MV: VP64-dCas9-VP64+MCP-VP64

-VP64 dCas9 VP64- + MCP.VP64-

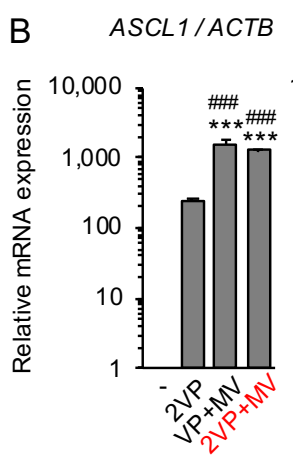

MYOD1/ACTB NEUROD1 / ACTB

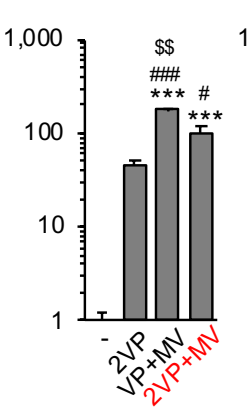

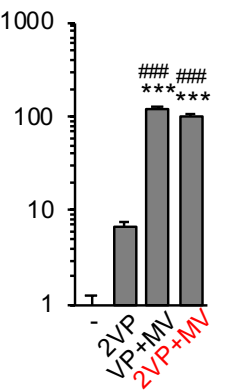

Figure 2. Analysis of CRISPRa-VP64 with addition of N-terminal VP64. (A) Schematic diagrams of the CRISPRa-VP64 systems tested; 2VP+MV is novel. (B) RNA expression of three endogenous genes was assayed in human HEK293T cells expressing the different CRISPRa systems. VP+MV showed the highest activity. Error bars indicate the mean $\pm \mathrm{SE}(\mathrm{n}=3)$. Statistical analysis was performed using one-way ANOVA with Tukey's multiple comparisons test. ${ }^{*}, P<0.05 ;^{* *}, P<0.01$; ${ }^{* * *}, P<0.005$ vs. non-induced

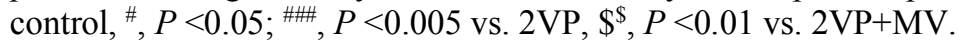

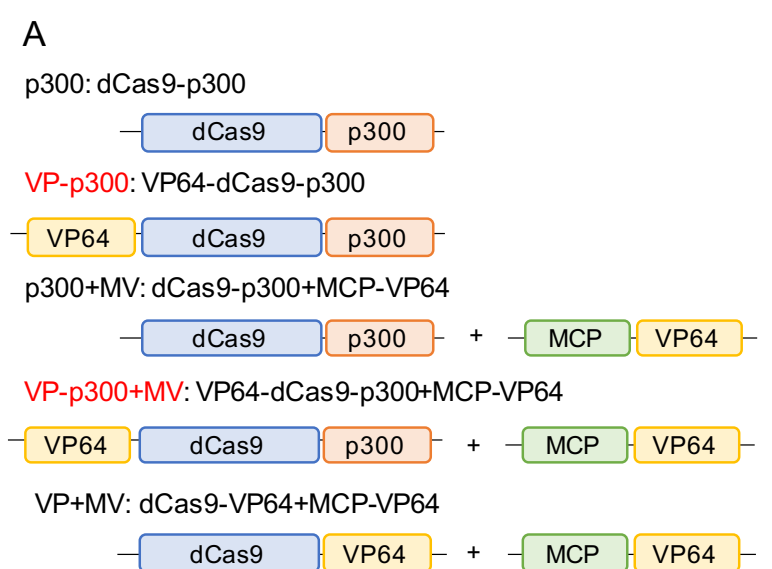

B
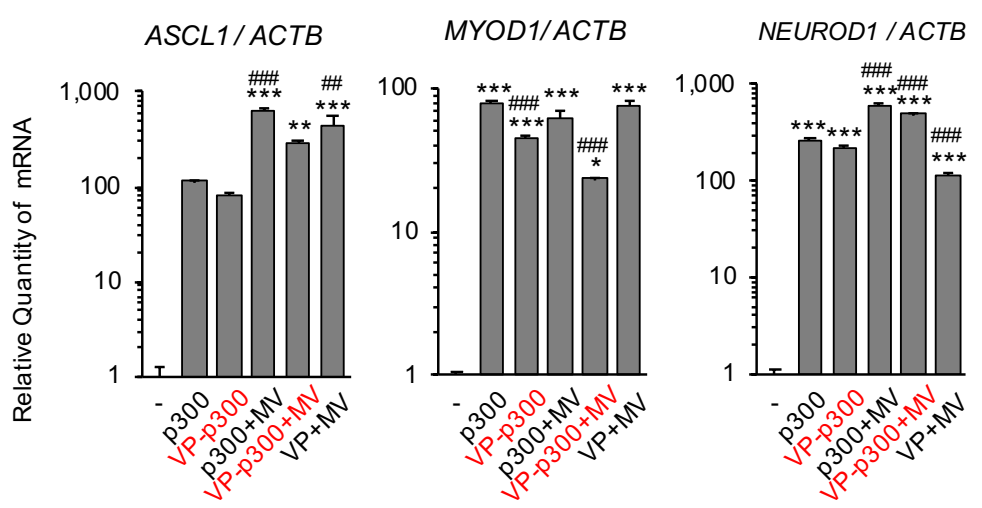

Figure 3. Analysis of combined VP64 and dCas9-p300 CRISPRa systems. (A) Schematic diagrams of the CRISPRa systems evaluated. VP-p300 and VP-p300+MV are novel. (B) RNA expression of three endogenous genes were tested in human HEK293T cells transfected with the indicated CRISPRa systems. The addition of VP64 to p300 by MS2-MCP scaffolding (to make p300+MV) enhanced transcriptional activation vs. p300 alone. Direct fusion of VP64 to the N-terminus of dCas9 (to make VP-p300) reduced the activity of transcriptional induction by p300 alone. Addition of VP64 to dCas9-p300 by MS2-MCP-VP64 (to make p300+MV) was more advantageous than direct fusion of VP64 (to make VP-p300+MV and VP-p300+MV). Error bars indicate the mean \pm SE (n=3). Statistical analysis was performed using one-way ANOVA with Tukey's multiple comparisons test. ${ }^{*}, P<0.05 ;^{* *}, P<0.01 ;{ }^{* * *}, P$ $<0.005$ vs. control, ${ }^{\#}, P<0.01$; ${ }^{\# \#}, P<0.005$ vs. p300.

\section{Combining the VP64 and dCas9-p300 systems}

Next, we combined the p300 system with N-terminal VP64 and MCP-VP64 (Figure 3A). The combination of p300 with MV enhanced the transcriptional activation of $A S C L 1$ and NEUROD1 by $\mathrm{p} 300$ alone. Transcriptional

activation of MYOD1 was equivalent in p300 and $\mathrm{p} 300+\mathrm{MV}$. On the other hand, the addition of N-terminal VP64 decreased transcriptional induction for both p300 and p300+MV (Figure 3B).

\section{Multiplexing of gRNAs targeting single genes}

Multiplexing of gRNAs targeting single genes has been shown to enhance transcriptional activation by dCas9VP64 ${ }^{89}$ and other CRISPRa systems ${ }^{25}$. Here we focused on $2 \mathrm{VP}, \mathrm{VP}+\mathrm{MV}$, and $\mathrm{p} 300+\mathrm{MV}$ (Figure 4A), which showed high activity with single gRNAs (Figure 2B and $3 \mathrm{~B}$ ), and compared them in the context of multiple gRNAs. $A S C L 1, M Y O D 1$, and $I L 1 R N^{14}$, for which several active gRNAs have already been identified, were used as representative genes. gRNA multiplexing enhanced $A S C L 1, M Y O D 1$, and $I L 1 R N$ expression compared with single gRNAs. Similar to the single gRNA studies, $\mathrm{VP}+\mathrm{MV}$ and $\mathrm{p} 300+\mathrm{MV}$ showed higher activity than $2 \mathrm{VP}$ with multiplexed gRNAs (Figure 4C). Further analyses showed that the enhanced activity of $\mathrm{VP}+\mathrm{MV}$ and p300+MV by gRNA multiplexing was comparable to that of SAM with single gRNA expression (Figure 5).

\section{Activation of exogeneous reporter genes}

Finally, we utilized minimal CMV ${ }^{2}$ and TRE3G ${ }^{26}$ promoters, which have low basal activities, as targets for transcriptional activation of exogenous genes by CRISPRa. As shown in Figure 6B and 7B, we generated four reporter gene constructs: minimal CMV-TdTomato and -NanoLuc with one gRNA binding site; and TRE3GTdTomato and -NanoLuc with seven identical gRNA 


\section{A}

B

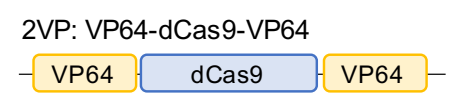

VP+MV: dCas9-VP64+MCP-VP64

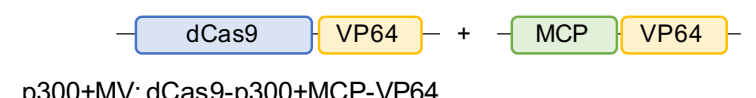

p300+MV: dCas9-p300+MCP-VP64
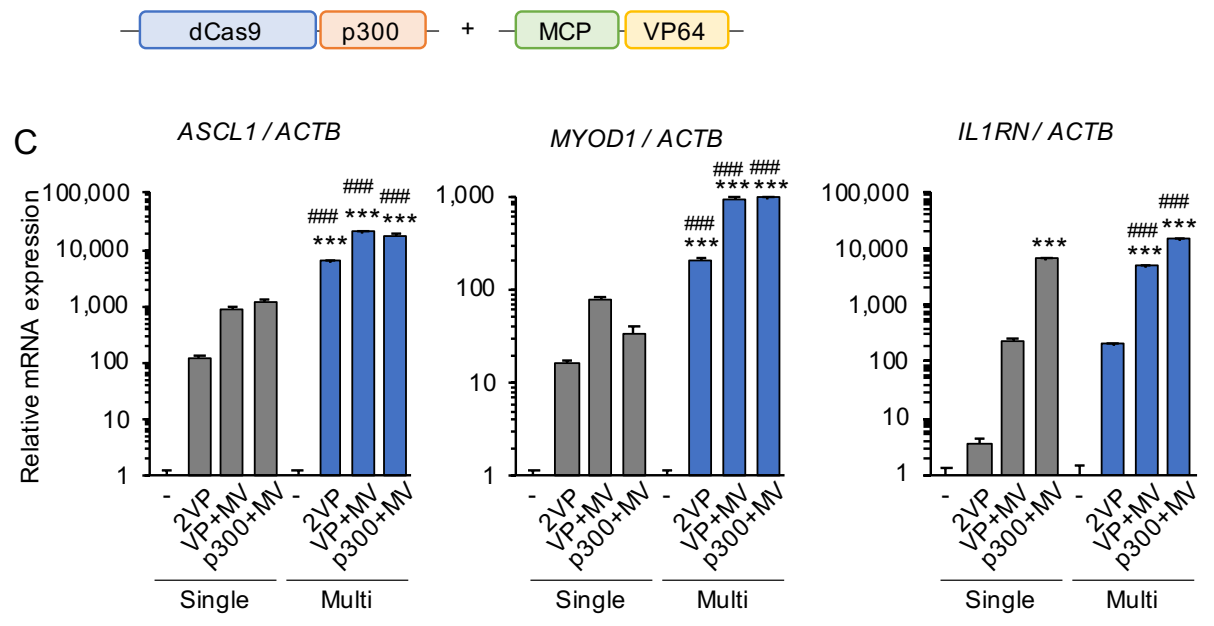

Figure 4. Analysis of the impact of multiplexing gRNAs targeting a single gene.

(A) Schematic diagrams of CRISPRa systems used for gRNA multiplexing to target a single gene. (B) Schematic diagrams showing the positions of single and multiple gRNAs near the transcriptional start site. (C) Expression of three endogenous genes in the presence of a single gRNA or multiple gRNAs in human HEK293T cells. Multiplexed gRNAs enhanced transcription in all three systems tested. Error bars indicate the mean $\pm \operatorname{SE}(n=3)$. Statistical analysis was performed using one-way ANOVA with Tukey's multiple comparisons test. ${ }^{* * *}$, $P<0.005$ vs. control, ${ }^{\#}, P<0.005$ vs. the level observed for each corresponding single gRNA experiment.

binding sites. Therefore, the TRE3G reporters should exhibit higher sensitivity to activation than the CMV reporters. None of these constructs showed reporter protein expression at baseline, but CRISPRa expression induced reporter protein expression (Figure 6C, 7C).

Similar to the activation of endogenous genes, VP+MV and p300+MV significantly induced both tdTomato and NanoLuc expression (Figure 6C, 7C). For the TRE3G reporter, with seven gRNA binding sites, the enhancement of reporter protein expression by $\mathrm{p} 300+\mathrm{MV}$ was limited compared to $\mathrm{VP}+\mathrm{MV}$. The spatial constraints of p300, a relatively large protein, could be responsible for this surprising finding.

\section{DISCUSSION}

CRISPR/Cas9-mediated transcriptional regulation has been used in broad research fields, including direct cell reprogramming ${ }^{1-3}$, disease modeling ${ }^{4}$, and therapeutic application ${ }^{5,6}$. Activation of endogenous genes by CRISPRa is a powerful tool for investigating cell biological processes requiring complex genetic regulation because of its ability to control multiple genes simultaneously. Moreover, the size of the target gene is not a limiting factor. Such technologies are important for hypothesis testing in the omics era ${ }^{27-30}$.
The present study aimed to compare dCas9-VP64 systems in the same contexts with the same parameters, including cell lines, transfection conditions, and target genes. Also, we used the highly active SAM and SPH CRISPRa systems as standards to compare the relative activities of the various CRISPRa-VP64 constructs. As the initial comparative study showed (Figure 1), all of the nextgeneration CRISPRa constructs were more active vs. the first generation dCas9-VP64, and SAM and SPH were the most active. These results are consistent with previous reports ${ }^{1}$, 10-12, which validates our comparative experimental system.

In addition to comparative studies of existing systems, we rationally designed new versions of CRISPRa-VP64 with different modes of VP64 recruitment. Our strategies were as follows: 1, addition of VP64 to the $\mathrm{N}$-terminus of $\mathrm{dCas} 9$ in dCas9-VP64+MCP-VP64; and 2, combination of VP64 and dCas9-p300 systems by either direct fusion or MS2-MCP scaffold. The addition of VP64 to the N-terminus of dCas9-VP64 had already been shown to increase the efficiency of transcriptional activation $^{1}$, but it was unclear whether the same effect would be observed for dCas9-VP64+MCP-VP64. Although the combination of MCP-VP64 with dCas9p300 enhances transcriptional activation when targeted to enhancers ${ }^{31}$, transcriptional activation when targeted to promoter regions has not been well studied. In addition, there were no studies on whether the addition of VP64 to the N-terminus of dCas9-p300 would enhance activity as it does for dCas9-VP64.

Our attempts to further improve dCas9-VP64+MCPVP64 and dCas9-p300+MCP-VP64 by adding N-terminal VP64 were unsuccessful. Direct fusion of VP64 to the Nterminus of dCas9 was effective for dCas9-VP64, which has only a single VP64, but our results suggest that adding one VP64 is not effective for dCas9-VP64+MCP-VP64, which already has an amplified number of VP64s via the MS2-MCP scaffold. Contrary to our expectations, fusion of VP64 to the N-terminus of dCas9-p300 resulted in decreased activity compared to that observed with dCas9p300 alone. Considering that the addition of VP64 by MV 


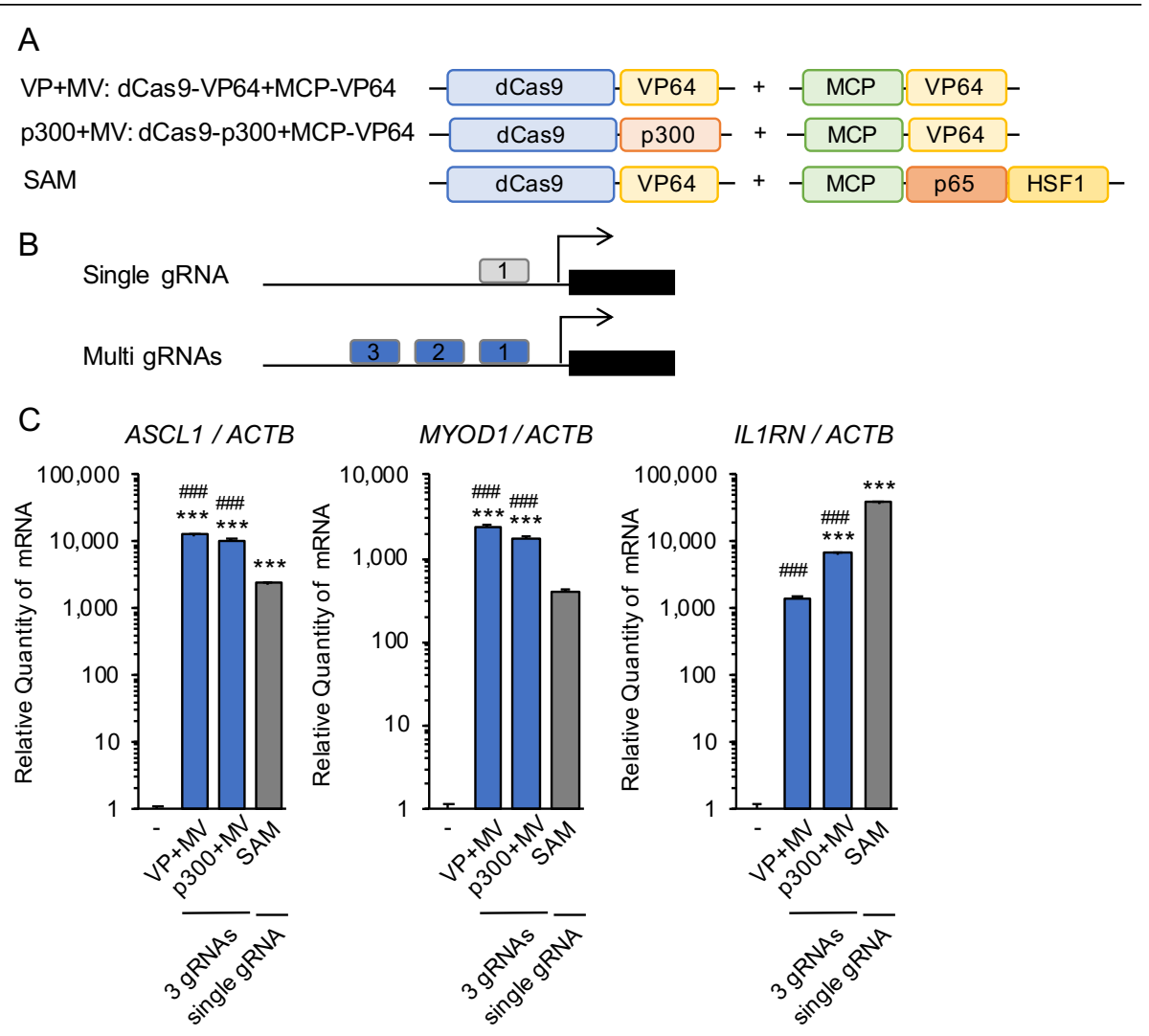

Figure 5. Comparison of multiplexing gRNAs for CRISPRa-VP64 and -p300 vs. single gRNA-targeted SAM. (A) Schematic diagrams of the CRISPRa systems used. (B) Schematic diagrams showing the positions of single and multiple gRNAs near the transcriptional start site. (C) Expression of three endogenous genes were tested in CRISPRa-transfected human HEK293T cells. VP+MV and p300+MV with multiple gRNAs showed higher ASCL1 and MYOD1 activation than SAM with a single gRNA. Error bars indicate the mean $\pm \mathrm{SE}(\mathrm{n}=3)$. Statistical analysis was performed using oneway ANOVA with Tukey's multiple comparisons test. ${ }^{* * *}, P<0.005$ vs. control, ${ }^{\# \#}, P$ $<0.005$ vs. SAM.

the TRE3G promoter. On the other hand, dCas9-p300+MCP-VP64, which has the largest size, showed only limited increases in activity with increased numbers of binding sites in the TRE3G promoter compared to other systems.

Multiplexing gRNAs targeting a single gene enhances transcriptional activation in various CRISPRa platforms. Chavez A et al. demonstrated that combining multiple gRNAs enhanced target gene expression in the dCas9-VP64, VPR, SAM and Suntag systems ${ }^{19}$, but other dCas9-VP64 systems were not tested. In the present study we carefully compared VP64dCas9-VP64, dCas9-VP64+MCPVP64 and dCas9-p300+MCP-VP64 systems in the presence of both a single gRNA and multiplexed gRNAs targeting single endogenous genes and exogenous genes. The results indicated that dCas9-VP64+MCP-VP64 and dCas9-p300+MCP-VP64 exhibit higher and more stable activity than VP64dCas9-VP64 whether single or multiple gRNAs were used (Figure 4). However, as mentioned above, dCas9p300+MCP-VP64 has spatial limitations, and the size of the coding sequence is larger than that of other CRISPRa constructs. If enhancer control is not the goal, VP64-dCas9VP64 and dCas9-VP64+MCP-VP64

on p300 additively enhanced transcriptional induction of ASCL1 and NEUROD1, N-terminal VP64 addition may decrease the ability of the dCas 9 complex to access the target site, either by increasing its size or changing protein conformation. The importance of such spatial accessibility has been reported regarding the incorporation of the DNA demethylase Tet1-CD for CRISPRa using the Suntag system. ${ }^{32}$ Since the p300 core domain is a relatively large protein domain, like Tet1-CD, spatial accessibility is likely to be involved.

Our results also demonstrated that when targeting a region of a single gene with multiple gRNAs, it is necessary to consider the spatial accessibility of the gRNAs due to the spacing of their target sequences. We were able to evaluate the enhancement of activity by gRNA multiplexing in exogenous genes by utilizing the miniCMV promoter reporter, which has only one gRNA binding site, and the TRE3G promoter reporter, which has seven gRNA binding sites (Figure 5, 6). dCas9-VP64, VP64-dCas9-VP64, and dCas9-VP64+MCP-VP64 showed increased activity with increased target sites in are easier to handle for packaging into a single lentivirus and for assembling a transgenic construct.

\section{CONCLUSION}

Based on our comparative analysis, dCas9-VP64 and p300 systems were significantly improved by combining with MCP-VP64, but not by direct fusion of VP64 to the N-terminus of dCas9. Moreover, we showed that gRNA multiplexing further enhanced target gene expression in dCas9-VP64+MCP-VP64 and dCas9-p300+MCP-VP64. Thus, our findings support the improvement of the dCas9VP64 system and contribute to developing a versatile and efficient CRISPRa platform.

\section{METHODS \\ Cell culture}

HEK293T cells were maintained in Dulbecco's Modified Eagle Medium (DMEM) (Gibco, \#11885092) supplemented with $10 \%$ heat inactivated fetal bovine serum (FBS) (Gibco, \#26140079) and 1\% penicillin/streptomycin at $37^{\circ} \mathrm{C}$ in a humidified $5 \% \mathrm{CO} 2$ 

available under aCC-BY-NC-ND 4.0 International license.

\begin{abstract}
A
VP: dCas9-VP64

2VP: VP64-dCas9-VP64

VP+MV: dCas9-VP64+MCP-VP64

P300+MV: dCas9-p300+MCP-VP64
\end{abstract}

B

Sensitivity: low miniCMV
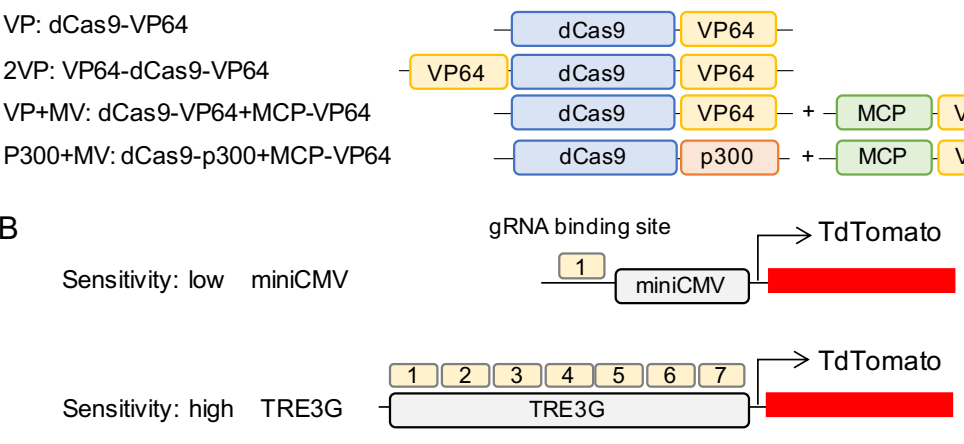

C Control
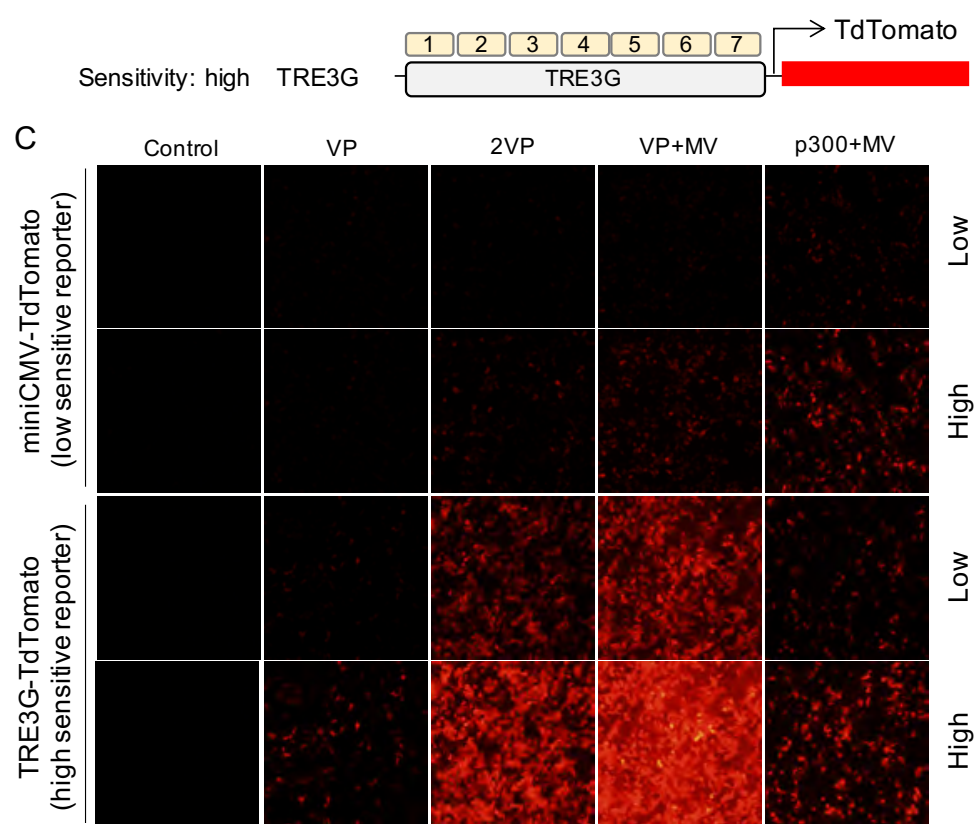

2VP

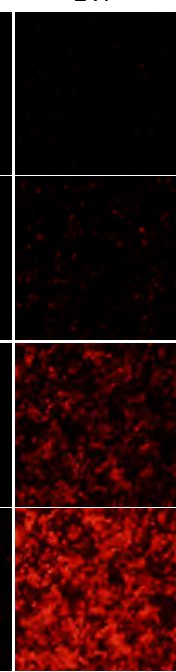

$V P+M V$

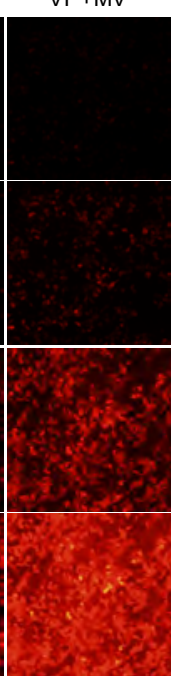

p300+MV

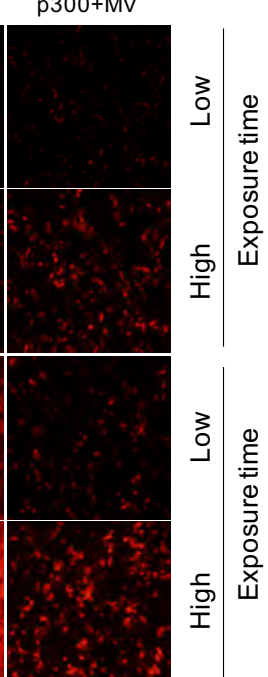

Figure 6. CRISPRa-mediated transcriptional activation of exogenous TdTomato reporter constructs. (A) Schematic diagrams of the CRISPRaVP64 and -p300 systems used. (B) Schematic diagrams of TdTomato reporter gene constructs. The miniCMV-driven TdTomato construct has one gRNA binding site, whereas the TRE3G-driven TdTomato construct has seven identical gRNA binding sites. (C) TdTomato fluorescence in HEK293T cells transfected with the indicated reporter and CRISPRa constructs. TdTomato expression was higher for TRE3G vs. miniCMV. The strength of TRE3G induction was in the order of $\mathrm{VP}+\mathrm{MV}>2 \mathrm{VP}>\mathrm{p} 300+\mathrm{MV}>\mathrm{VP}$.

incubator. Neuro-2a cells were maintained in Minimum Essential Media (MEM) (Gibco, \#11095080) supplemented with $10 \%$ heat inactivated fetal bovine serum (FBS) (Gibco, \#26140079) and 1\% penicillin/streptomycin at $37^{\circ} \mathrm{C}$ in a humidified $5 \% \mathrm{CO} 2$ incubator. For transfecting cells, Lipofectamine 3000 (Invitrogen, \#L3000015) was used by following the manufacturer's protocol. Briefly, 120,000 -150,000 cells per 12-well plate were seeded. After 20-24 h, cells were transfected with $1 \mu \mathrm{g}$ of total plasmid DNA, $2 \mu \mathrm{L}$ of $\mathrm{p} 3000$ reagent and $3 \mu \mathrm{L}$ of Lipofectamine 3000 reagent in $50 \mu \mathrm{L}$ of Opti-MEM (Gibco, \#31985070). At 48 h post transfection, transfected cells were used for q-RT-PCR analysis, fluorescence imaging and luciferase assays.

\section{Plasmid construction}

The plasmids used in this study are listed in Supplemental Table 1.

lenti dCAS-VP64_Blast (Addgene plasmid \#61425), lentiMP $\bar{H}$ v2 (Addgene plasmid \#89308) and lenti sgRNA(MS2) puro backbone (Addgene plasmid \#73795) ${ }^{11}$ were gifts from Feng Zhang. ${ }^{11,} 33$ Lenti_MCPVP64_Hygro (Addgene plasmid \#138458) was a gift from Jian $\mathrm{Xu}^{31}$ EF1 $\alpha$-dCas910xGCN4_Hygro and EF1 $\alpha$-scFv-p65-HSF1Blast was generated by Gibson assembly cloning (NEB, \#E5520S). Briefly, LentidCas9-10xGCN4_Hygro and Lenti-scFv-p65HSF_Blast were amplified from dSV40-NLSdCas9-HA-NLS-NLS-10xGCN4 (Addgene plasmid \#107310, gifted from Hui Yang) and

EF1 $\alpha$-scFv-p65-HSF1-T2A-EGFP-WPREPolyA (Addgene plasmid \#107311, a gift from Hui Yang) $)^{12}$ and inserted into lentiMPH v2 and lenti dCAS-VP64_Blast backbone respectively. Lenti-EF1 $\alpha$-dCas9-p300_Blast was generated by replacing the PuroR of pLV-dCas9-p300P2A-PuroR (Addgene plasmid \#83889, a gift from Charles Gersbach) ${ }^{34}$ with BlastR using Gibson Assembly. Lenti-EF1 $\alpha$-VP64-dCas9VP64_Blast was generated by inserting VP64 in-frame right after the ATG start codon of lenti dCAS-VP64_Blast (Addgene plasmid \#61425). Similarly, Lenti-EF1 $\alpha$-VP64-dCas9p300_Blast was generated by inserting VP64 in-frame right after the ATG start codon of Lenti-EF1 $\alpha$-dCas9-p300_Blast. Lenti-EF1 $\alpha$ scFv-VP64_Blast was generated by fusing scFv-sfGFP-GB1 from pHRdSV40_scFv_GCN4_sfGFP_p65hsf1_GB1_NLS (Addgene plasmid \#79372, a gift from George Church) ${ }^{19}$ and VP64 from lenti dCAS-VP64_Blast (Addgene plasmid \#61425) using Gibson Assembly and inserting into the backbone of lenti dCAS-VP64_Blast (Addgene plasmid \#61425). pCR-U6-gRNA-miniCMV-TdTomato was generated by inserting miniCMV-TdTomato from reporter-gT1 (Addgene plasmid \#47320, a gift from George Church) ${ }^{35}$ into lenti sgRNA(MS2)_puro backbone (Addgene plasmid \#73795); then U6-gRNAminiCMV-TdTomato was inserted into pCR Blunt IITOPO. pCR-U6-gRNA-miniCMV-Nluc was generated by replacing TdTomato with Nluc from pNLF1-C [CMV/Hygro] (Promega \#N1361) using Gibson Assembly. pCR-U6-gRNA-TRE3G-TdTomato and-Nluc were generated by replacing the miniCMV promoter of lenti sgRNA(MS2)_puro-miniCMV-TdTomato/Nluc with the TRE3G promoter from pTRE3G (Takara, \#631173) using Gibson Assembly and inserting into pCR Blunt II-TOPO. Plasmids which were newly generated in the present study will be deposited into Addgene. 

available under aCC-BY-NC-ND 4.0 International license.

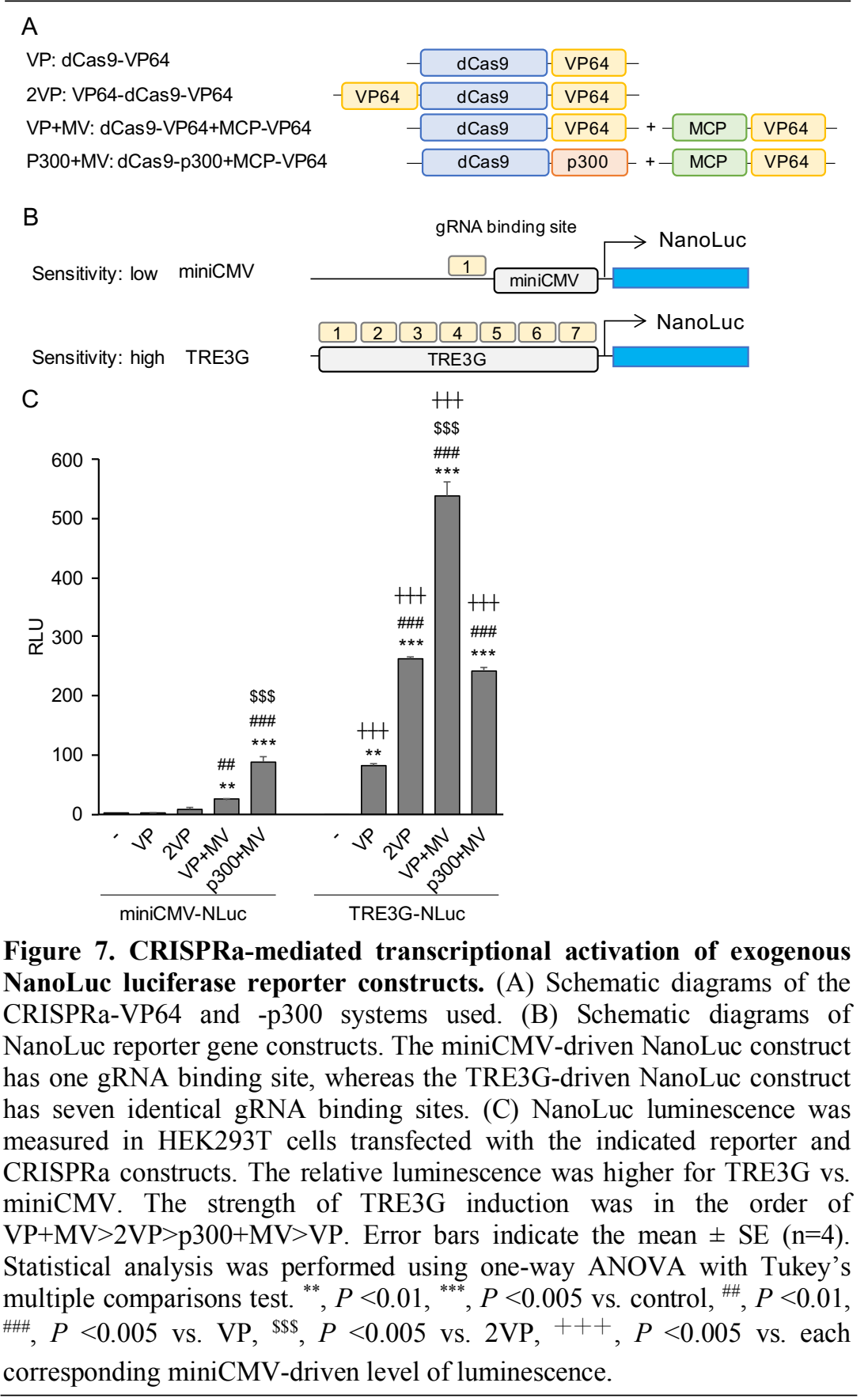

vector (80ng), $1 \mu \mathrm{L}$ of Esp3I restriction enzyme (NEB, \#R0734S), $1.5 \mu \mathrm{L}$ of T4 DNA ligase (NEB, \#M0202S), $2 \mu \mathrm{L}$ 10x T4 DNA ligase buffer and $12.5 \mu \mathrm{L}$ of nuclease free water. The Golden Gate Assembly reaction was performed in a thermal cycler using the following program: Step 1: $37{ }^{\circ} \mathrm{C}$ for $5 \mathrm{~min}$; Step 2: $16^{\circ} \mathrm{C}$ for $5 \mathrm{~min}$; repeat steps $1-2$ for 60 cycles; step $3: 75^{\circ} \mathrm{C}$ for $5 \mathrm{~min}$, step $4: 4^{\circ} \mathrm{C}$ hold. $5 \mu \mathrm{L}$ of reaction was transformed into NEB 5-alpha Competent $E$. coli (NEB, \#C2987H) by following the manufacturer's protocol.

\section{RNA extraction and qRT-PCR analysis}

Cells were harvested $48 \mathrm{~h}$ post-transfection. Transfected cells were lysed with TRIzol reagent (Invitrogen, \#15596018) and RNA was extracted and purified by following the manufacturer's protocol. Purified RNA was quantified by A260/280 absorbance. cDNA was synthesized using the PrimeScript RT Master Mix (Takara, \#RR036A) by following the manufacturer's protocol. Briefly, cDNA was synthesized using $62.5 \mathrm{ng}$ of RNA per target gene at $37{ }^{\circ} \mathrm{C}$ for $30 \mathrm{~min}$, and then RT enzyme was heat inactivated at $85^{\circ} \mathrm{C}$ for $5 \mathrm{sec}$. For qPCR analysis, Fast SYBR Green Master Mix (Applied Biosystems, \#4385612) was used by following the manufacturer's protocol. The sequences of primers used for qRT-PCR are listed in Supplemental Table 1.

\section{Luciferase assay}

The procedure of dual luciferase assay was described previously ${ }^{36}$. Briefly, pCR4-U6gRNA-miniCMV-NanoLuc or pCR4-U6gRNA-TRE3G-NanoLuc and HSV-TK-Luc2 plasmids were transfected into HEK293T cells. At $48 \mathrm{~h}$ post transfection, transfected cells were washed once with phosphate buffer saline. Then, ONE-Glo Ex reagent was added, and firefly

\section{gRNA sequence and cloning}

The sequences of gRNAs used for activating endogenous and exogenous genes are listed in Supplemental Table 1. Oligonucleotides of 5'CACC-sense gRNA-3' and 5', AAAC-antisense gRNA-3' were purchased from Integrated DNA Technology (IDT) and they were annealed by cooling from $95^{\circ} \mathrm{C}$ to $25^{\circ} \mathrm{C}$ for 1.5 hours. Annealing reaction mixtures were prepared as follows: $1 \mu \mathrm{L}$ of sense oligo $(100 \mu \mathrm{M}), 1 \mu \mathrm{L}$ of antisense oligo $(100 \mu \mathrm{M}), 10 \mathrm{x}$ annealing buffer $(400 \mathrm{mM}$ Tris- $\mathrm{HCl}(\mathrm{pH}$ 8.0); $200 \mathrm{mM} \mathrm{MgCl} 2 ; 500 \mathrm{mM} \mathrm{NaCl})$ and $7 \mu \mathrm{L}$ of nuclease free water. Then, annealed oligo was cloned into gRNA expression vector by Golden Gate Assembly.

Golden Gate Assembly reactions were prepared as follows: $2.5 \mu \mathrm{L}$ of annealed oligo, $1 \mu \mathrm{L}$ of gRNA cloning luciferase was measured. Subsequently, NanoDLR Stop $\&$ Go reagent (Promega, \#N1620) was added, and the reaction plate was incubated for $10 \mathrm{~min}$ at room temperature, then NanoLuc luciferase was measured. The luciferase activity in the cell lysates was measured using a GloMax Navigator system (Promega). All luciferase assays were conducted in LumiNunc 96-well white plates (Thermo Scientific, \#136101). NanoLuc luciferase was normalized by constitutively expressed firefly luciferase.

\section{Statistics}

Statistical parameters are reported in the Figure Legends. Gene expression analysis was performed in triplicate using 3 independent transfections. Luciferase assays were 
performed in quadruplicate using 4 independent cell cultures. The significance of differences in multiplegroups was determined by analysis of variance (ANOVA) with Tukey-Kramer post-hoc or Dunnett's tests. Differences with $P$ values of less than 0.05 were considered statistically significant.

\section{AUTHOR INFORMATION \\ Corresponding Author}

Jeffrey H. Miner - Division of Nephrology, MSC-8126012-0853, Washington University School of Medicine, 4523 Clayton Ave., St. Louis, Missouri, 63110, USA; orcid.org/0000-0002-1510-8714

Email: minerj@wustl.edu

\section{Author}

Kohei Omachi - Division of Nephrology, MSC-8126012-0853, Washington University School of Medicine, 4523 Clayton Ave., St. Louis, Missouri, 63110, USA.

\section{Author Contributions}

K.O designed the study, performed experiments, and wrote the manuscript. J.H.M designed the study and edited the manuscript.

\section{ACKNOWLEDGEMENTS}

This work was supported by grants from the National Institutes of Health (R01DK058366 and R01DK078314 to J.H.M.), the Children's Discovery Institute of Washington University and St. Louis Children's Hospital (to J.H.M.), and the Japan Society for the Promotion of Science (JSPS) Program for Postdoctoral Fellowships for Research Abroad (to K.O.).

\section{AUTHOR DISCLOSURE STATEMENT}

The authors declare no competing financial interests.

\section{REFERENCES}

1. Chakraborty, S.; Ji, H.; Kabadi, A. M.; Gersbach, C. A.; Christoforou, N.; Leong, K. W., A CRISPR/Cas9-based system for reprogramming cell lineage specification. Stem Cell Reports 2014, 3 (6), 940-7.

2. Chavez, A.; Scheiman, J.; Vora, S.; Pruitt, B. W.; Tuttle, M.; E, P. R. I.; Lin, S.; Kiani, S.; Guzman, C. D.; Wiegand, D. J.; Ter-Ovanesyan, D.; Braff, J. L.; Davidsohn, N.; Housden, B. E.; Perrimon, N.; Weiss, R.; Aach, J.; Collins, J. J.; Church, G. M., Highly efficient Cas9-mediated transcriptional programming. Nat Methods 2015, 12 (4), 326-8.

3. Black, J. B.; Adler, A. F.; Wang, H. G.; D'Ippolito, A. M.; Hutchinson, H. A.; Reddy, T. E.; Pitt, G. S.; Leong, K. W.; Gersbach, C. A., Targeted Epigenetic Remodeling of Endogenous Loci by
CRISPR/Cas9-Based Transcriptional Activators Directly Converts Fibroblasts to Neuronal Cells. Cell Stem Cell 2016, 19 (3), 406-14.

4. $\quad$ Wangensteen, K. J.; Wang, Y. J.; Dou, Z.; Wang, A. W.; Mosleh-Shirazi, E.; Horlbeck, M. A.; Gilbert, L. A.; Weissman, J. S.; Berger, S. L.; Kaestner, K. H., Combinatorial genetics in liver repopulation and carcinogenesis with a in vivo CRISPR activation platform. Hepatology 2018, 68 (2), 663-676.

5. Kemaladewi, D. U.; Bassi, P. S.; Erwood, S.; Al-Basha, D.; Gawlik, K. I.; Lindsay, K.; Hyatt, E.; Kember, R.; Place, K. M.; Marks, R. M.; Durbeej, M.; Prescott, S. A.; Ivakine, E. A.; Cohn, R. D., A mutationindependent approach for muscular dystrophy via upregulation of a modifier gene. Nature 2019, 572 (7767), 125-130.

6. Matharu, N.; Rattanasopha, S.; Tamura, S.; Maliskova, L.; Wang, Y.; Bernard, A.; Hardin, A.; Eckalbar, W. L.; Vaisse, C.; Ahituv, N., CRISPRmediated activation of a promoter or enhancer rescues obesity caused by haploinsufficiency. Science 2019, 363 (6424).

7. Jinek, M.; Chylinski, K.; Fonfara, I.; Hauer, M.; Doudna, J. A.; Charpentier, E., A programmable dual-RNA-guided DNA endonuclease in adaptive bacterial immunity. Science 2012, 337 (6096), 816-21.

8. Perez-Pinera, P.; Kocak, D. D.; Vockley, C. M.; Adler, A. F.; Kabadi, A. M.; Polstein, L. R.; Thakore, P. I.; Glass, K. A.; Ousterout, D. G.; Leong, K. W.; Guilak, F.; Crawford, G. E.; Reddy, T. E.; Gersbach, C. A., RNA-guided gene activation by CRISPR-Cas9-based transcription factors. Nat Methods 2013, 10 (10), 973-6.

9. Maeder, M. L.; Linder, S. J.; Cascio, V. M.; Fu, Y.; Ho, Q. H.; Joung, J. K., CRISPR RNA-guided activation of endogenous human genes. Nat Methods 2013, 10 (10), 977-9.

10. Tanenbaum, M. E.; Gilbert, L. A.; Qi, L. S.; Weissman, J. S.; Vale, R. D., A protein-tagging system for signal amplification in gene expression and fluorescence imaging. Cell 2014, 159 (3), 635-46.

11. Konermann, S.; Brigham, M. D.; Trevino, A. E.; Joung, J.; Abudayyeh, O. O.; Barcena, C.; Hsu, P. D.; Habib, N.; Gootenberg, J. S.; Nishimasu, H.; Nureki, O.; Zhang, F., Genome-scale transcriptional activation by an engineered CRISPR-Cas 9 complex. Nature 2015, 517 (7536), 583-8.

12. Zhou, H.; Liu, J.; Zhou, C.; Gao, N.; Rao, Z.; Li, H.; Hu, X.; Li, C.; Yao, X.; Shen, X.; Sun, Y.; Wei, Y.; Liu, F.; Ying, W.; Zhang, J.; Tang, C.; Zhang, X.; Xu, H.; Shi, L.; Cheng, L.; Huang, P.; 

available under aCC-BY-NC-ND 4.0 International license.

Yang, H., In vivo simultaneous transcriptional activation of multiple genes in the brain using CRISPR-dCas9activator transgenic mice. Nat Neurosci 2018, 21 (3), 440-446.

13. Kunii, A.; Hara, Y.; Takenaga, M.; Hattori, N.; Fukazawa, T.; Ushijima, T.; Yamamoto, T.; Sakuma, T., Three-Component Repurposed Technology for Enhanced Expression: Highly Accumulable Transcriptional Activators via Branched Tag Arrays. CRISPR J 2018, 1, 337-347.

14. Hilton, I. B.; D'Ippolito, A. M.; Vockley, C. M.; Thakore, P. I.; Crawford, G. E.; Reddy, T. E.; Gersbach, C. A., Epigenome editing by a CRISPR-Cas9based acetyltransferase activates genes from promoters and enhancers. Nat Biotechnol 2015, 33 (5), 510-7.

15. Liu, X. S.; Wu, H.; Ji, X.; Stelzer, Y.; Wu, X.; Czauderna, S.; Shu, J.; Dadon, D.; Young, R. A.; Jaenisch, R., Editing DNA Methylation in the Mammalian Genome. Cell 2016, 167 (1), 233-247 e17.

16. Ewen-Campen, B.; Yang-Zhou, D.; Fernandes, V. R.; Gonzalez, D. P.; Liu, L. P.; Tao, R.; Ren, X.; Sun, J.; Hu, Y.; Zirin, J.; Mohr, S. E.; Ni, J. Q.; Perrimon, N., Optimized strategy for in vivo Cas9activation in Drosophila. Proc Natl Acad Sci US A 2017, 114 (35), 9409-9414.

17. Yamagata, T.; Raveau, M.; Kobayashi, K.; Miyamoto, H.; Tatsukawa, T.; Ogiwara, I.; Itohara, S.; Hensch, T. K.; Yamakawa, K., CRISPR/dCas9-based Scn la gene activation in inhibitory neurons ameliorates epileptic and behavioral phenotypes of Dravet syndrome model mice. Neurobiol Dis 2020, 141, 104954.

18. Platt, R. J.; Chen, S.; Zhou, Y.; Yim, M. J.; Swiech, L.; Kempton, H. R.; Dahlman, J. E.; Parnas, O.; Eisenhaure, T. M.; Jovanovic, M.; Graham, D. B.; Jhunjhunwala, S.; Heidenreich, M.; Xavier, R. J.; Langer, R.; Anderson, D. G.; Hacohen, N.; Regev, A.; Feng, G.; Sharp, P. A.; Zhang, F., CRISPR-Cas9 knockin mice for genome editing and cancer modeling. Cell 2014, 159 (2), 440-55.

19. Chavez, A.; Tuttle, M.; Pruitt, B. W.; EwenCampen, B.; Chari, R.; Ter-Ovanesyan, D.; Haque, S. J.; Cecchi, R. J.; Kowal, E. J. K.; Buchthal, J.; Housden, B. E.; Perrimon, N.; Collins, J. J.; Church, G., Comparison of Cas9 activators in multiple species. Nat Methods 2016, 13 (7), 563-567.

20. Vora, S.; Cheng, J.; Xiao, R.; VanDusen, N. J.; Quintino, L.; Pu, W. T.; Vandenberghe, L. H.; Chavez, A.; Church, G., Rational design of a compact CRISPRCas9 activator for AAV-mediated delivery. bioRxiv 2018, 298620.
21. Lu, J.; Zhao, C.; Zhao, Y.; Zhang, J.; Zhang, Y.; Chen, L.; Han, Q.; Ying, Y.; Peng, S.; Ai, R.; Wang, Y., Multimode drug inducible CRISPR/Cas9 devices for transcriptional activation and genome editing. Nucleic Acids Res 2018, 46 (5), e25.

22. Martella, A.; Firth, M.; Taylor, B. J. M.; Goppert, A.; Cuomo, E. M.; Roth, R. G.; Dickson, A. J.; Fisher, D. I., Systematic Evaluation of CRISPRa and CRISPRi Modalities Enables Development of a Multiplexed, Orthogonal Gene Activation and Repression System. ACS Synth Biol 2019, 8 (9), 19982006.

23. Kretzmann, J. A.; Evans, C. W.; Moses, C.; Sorolla, A.; Kretzmann, A. L.; Wang, E.; Ho, D.; Hackett, M. J.; Dessauvagie, B. F.; Smith, N. M.; Redfern, A. D.; Waryah, C.; Norret, M.; Iyer, K. S.; Blancafort, P., Tumour suppression by targeted intravenous non-viral CRISPRa using dendritic polymers. Chem Sci 2019, 10 (33), 7718-7727.

24. Petazzi, P.; Torres-Ruiz, R.; Fidanza, A.; Roca-Ho, H.; Gutierrez-Aguera, F.; Castano, J.; Rodriguez-Perales, S.; Diaz de la Guardia, R.; LopezMillan, B.; Bigas, A.; Forrester, L. M.; Bueno, C.; Menendez, P., Robustness of Catalytically Dead Cas9 Activators in Human Pluripotent and Mesenchymal Stem Cells. Mol Ther Nucleic Acids 2020, 20, 196-204.

25. Savell, K. E.; Bach, S. V.; Zipperly, M. E.; Revanna, J. S.; Goska, N. A.; Tuscher, J. J.; Duke, C. G.; Sultan, F. A.; Burke, J. N.; Williams, D.; Ianov, L.; Day, J. J., A Neuron-Optimized CRISPR/dCas9 Activation System for Robust and Specific Gene Regulation. eNeuro 2019, 6 (1).

26. Nakamura, M.; Srinivasan, P.; Chavez, M.; Carter, M. A.; Dominguez, A. A.; La Russa, M.; Lau, M. B.; Abbott, T. R.; Xu, X.; Zhao, D.; Gao, Y.; Kipniss, N. H.; Smolke, C. D.; Bondy-Denomy, J.; Qi, L. S., Anti-CRISPR-mediated control of gene editing and synthetic circuits in eukaryotic cells. Nat Commun 2019, 10 (1), 194.

27. Wang, G.; Chow, R. D.; Bai, Z.; Zhu, L.; Errami, Y.; Dai, X.; Dong, M. B.; Ye, L.; Zhang, X.; Renauer, P. A.; Park, J. J.; Shen, L.; Ye, H.; Fuchs, C. S.; Chen, S., Multiplexed activation of endogenous genes by CRISPRa elicits potent antitumor immunity. Nat Immunol 2019, 20 (11), 1494-1505.

28. Alda-Catalinas, C.; Bredikhin, D.; HernandoHerraez, I.; Santos, F.; Kubinyecz, O.; EckersleyMaslin, M. A.; Stegle, O.; Reik, W., A Single-Cell Transcriptomics CRISPR-Activation Screen Identifies Epigenetic Regulators of the Zygotic Genome Activation Program. Cell Syst 2020, 11 (1), 25-41 e9. 
29. Black, J. B.; McCutcheon, S. R.; Dube, S.;

Barrera, A.; Klann, T. S.; Rice, G. A.; Adkar, S. S.;

Soderling, S. H.; Reddy, T. E.; Gersbach, C. A., Master

Regulators and Cofactors of Human Neuronal Cell Fate Specification Identified by CRISPR Gene Activation Screens. Cell Rep 2020, 33 (9), 108460.

30. Tian, R.; Abarientos, A.; Hong, J.; Hashemi, S. H.; Yan, R.; Drager, N.; Leng, K.; Nalls, M. A.; Singleton, A. B.; Xu, K.; Faghri, F.; Kampmann, M., Genome-wide CRISPRi/a screens in human neurons link lysosomal failure to ferroptosis. Nat Neurosci 2021, 24 (7), 1020-1034.

31. Li, K.; Liu, Y.; Cao, H.; Zhang, Y.; Gu, Z.; Liu, X.; Yu, A.; Kaphle, P.; Dickerson, K. E.; Ni, M.; $\mathrm{Xu}$, J., Interrogation of enhancer function by enhancertargeting CRISPR epigenetic editing. Nat Commun 2020, 11 (1), 485 .

32. Morita, S.; Noguchi, H.; Horii, T.;

Nakabayashi, K.; Kimura, M.; Okamura, K.; Sakai, A.; Nakashima, H.; Hata, K.; Nakashima, K.; Hatada, I., Targeted DNA demethylation in vivo using dCas9peptide repeat and scFv-TET1 catalytic domain fusions. Nat Biotechnol 2016, 34 (10), 1060-1065.

33. Joung, J.; Konermann, S.; Gootenberg, J. S.; Abudayyeh, O. O.; Platt, R. J.; Brigham, M. D.; Sanjana, N. E.; Zhang, F., Genome-scale CRISPR-Cas9 knockout and transcriptional activation screening. Nat Protoc 2017, 12 (4), 828-863.

34. Klann, T. S.; Black, J. B.; Chellappan, M.; Safi, A.; Song, L.; Hilton, I. B.; Crawford, G. E.; Reddy, T. E.; Gersbach, C. A., CRISPR-Cas9 epigenome editing enables high-throughput screening for functional regulatory elements in the human genome. Nat Biotechnol 2017, 35 (6), 561-568.

35. Mali, P.; Aach, J.; Stranges, P. B.; Esvelt, K. M.; Moosburner, M.; Kosuri, S.; Yang, L.; Church, G. M., CAS9 transcriptional activators for target specificity screening and paired nickases for cooperative genome engineering. Nat Biotechnol 2013, 31 (9), 833-8.

36. Omachi, K.; Kai, H.; Roberge, M.; Miner, J. $\mathrm{H}$., Aminoglycoside-induced premature termination codon readthrough of COL4A5 nonsense mutations that cause Alport syndrome. bioRxiv 2021, 2021.06.11.448099. 